\title{
Scleral pits represent degeneration around the posterior ciliary arteries and are signs of disease severity in choroideremia
}

\author{
Christy M. Harvey $y^{1,2} \cdot$ S. Scott Whitmore ${ }^{1,2} \cdot$ D. Brice Critser ${ }^{1,2} \cdot$ Louisa M. Affatigato $^{1,2} \cdot$ Heather T. Daggett $^{1,2} \cdot$ \\ Edwin M. Stone ${ }^{1,2} \cdot \operatorname{lan}$ C. Han $\mathbb{1}^{1,2}$
}

Received: 4 June 2019 / Revised: 11 August 2019 / Accepted: 1 September 2019 / Published online: 25 September 2019

(c) The Author(s), under exclusive licence to The Royal College of Ophthalmologists 2019

\begin{abstract}
Background Choroideremia is an X-linked recessive condition characterized by progressive chorioretinal degeneration. Recently, peculiar scleral ectasias, termed scleral "pits" and "tunnels," have been described as a novel finding in patients with choroideremia, but little is known regarding their etiology or their evolution over time.

Subjects This is a retrospective chart review of consecutive patients with molecularly-confirmed choroideremia and related female carriers seen at a university-based tertiary referral center from January 2010 to July 2016. Multimodal imaging was evaluated for the evolution of scleral pits on fundus photography and scleral tunnels on optical coherence tomography (OCT). The presence of scleral pits and tunnels was correlated with markers of disease severity including age, visual acuity, and severity of visual field loss.

Results Thirty patients ( 21 affected males, 9 female carriers) were included in the study. Scleral pits were seen in $38.1 \%$ ( $8 /$ 21) of affected males and found to occur at insertion sites of the posterior ciliary arteries. Those with scleral pits were older, had poorer visual acuity, and more severe visual field loss than those without $(p \leq 0.05)$. Scleral tunnels were common (68.4\%, $13 / 19$ affected males with available OCT imaging), but no statistically-significant associations with disease severity were seen. The development of new scleral pits and tunnels was observed on longitudinal imaging in 4 and 2 affected males, respectively. No scleral pits or tunnels were visualized in any female carriers.

Conclusions Scleral pits represent degeneration around the posterior ciliary arteries and may be useful as clinical markers of disease severity in choroideremia.
\end{abstract}

\section{Introduction}

Choroideremia is an $\mathrm{X}$-linked recessive chorioretinal degeneration characterized by progressive loss of the photoreceptors, retinal pigment epithelium (RPE), and choriocapillaris $[1,2]$. The causative gene, CHM, was first described by Cremers et al. in 1990 and consists of 15 exons with a large range in size [1, 3]. CHM encodes Rab Escort Protein 1 (REP-1), a ubiquitously-expressed protein thought to be involved in vesicular trafficking [1]. The symptoms of

Ian C. Han

ian-han@uiowa.edu

1 Department of Ophthalmology and Visual Sciences, Carver College of Medicine, University of Iowa, Iowa City, IA, USA

2 Institute for Vision Research, University of Iowa, Iowa City, IA, USA choroideremia include nyctalopia and peripheral visual field loss, with decreased central vision developing much later in the disease course [4]. Affected male patients have a characteristic fundus appearance including confluent, scalloped areas of RPE atrophy, and choriocapillaris loss, which typically starts in the mid-periphery and progresses centripetally. Female carries of this condition have a widely variable phenotype due at least in part to random $\mathrm{X}$ chromosome inactivation [1].

Recently, peculiar scleral ectasias, termed scleral "pits" and "tunnels," have been described in patients with choroideremia [5, 6]. These scleral excavations are often visible on funduscopic examination and spectral-domain optical coherence tomography (OCT) imaging shows them to be associated with interruptions of the RPE, choroid, and sclera. Patients with these anomalies tend to be older and have poorer visual acuity, suggesting that they may be signs of retinal degeneration [5], but to date, there have been no reports detailing their evolution over time. 
In this study, we seek to expand our understanding of scleral pits and tunnels by evaluating longitudinal fundus photography and spectral-domain OCT imaging in a cohort of patients with molecularly-confirmed choroideremia and related female carriers. We evaluate the prevalence of visible scleral excavations and relate their presence to measures of disease severity, including visual acuity and degree of visual field loss. We further characterize the regional distribution of scleral ectasias in the posterior pole by co-registering their location on multimodal imaging to demonstrate their association with surrounding structures including the posterior ciliary arteries. Finally, we relate our findings to existing literature to generate hypotheses as to their etiology and role in the pathophysiology of choroideremia.

\section{Methods}

This is a retrospective chart review of consecutive patients with molecularly-confirmed choroideremia seen at the University of Iowa from January 2010 to July 2016 as part of a previously-published cohort [7]. The study adhered to the tenets of the Declaration of Helsinki and was approved by the Institutional Review Board of the University of Iowa Hospital and Health System. Demographic data including age, gender, and genotype were recorded. All genetic testing was performed through the Carver Nonprofit Genetic Testing Laboratory at the University of Iowa. For each clinic visit, best-corrected visual acuity and degree of refractive error (spherical equivalent) were extracted from the chart. Goldmann visual fields were assessed for diffuse field loss as defined by the complete absence of the I2e isopter, or severe central visual field loss as defined by a less than $20^{\circ}$ horizontal field to the V4e isopter centrally.

Multimodal imaging, including $50^{\circ}$ color fundus photographs (Topcon TRC-50DX, Topcon America, Paramus, New Jersey) and spectral-domain OCT (Heidelberg Spectralis; Heidelberg Engineering, Heidelberg, Germany) were reviewed for each patient. Macular volume scans were cantered on the fovea and included both $20^{\circ}$ volume scans (25 line scans, $6.2 \mathrm{~mm}$ horizontally per line scan, $512 \times 496$ pixels) and $30^{\circ}$ volume scans (61 line scans, $9.7 \mathrm{~mm}$ horizontally per line scan, $768 \times 496$ pixels). Scans taken at follow-up visits were registered to their initial fundus location using TruTrack Active Eye Tracking via the Heidelberg Spectralis software. Fundus photographs and OCTs were reviewed by two retina specialists as independent graders $(\mathrm{CMH}, \mathrm{ICH})$ for the presence of scleral pits and tunnels. To clarify the terminology in the literature, where scleral "pits" and "tunnels" are currently used interchangeably, we defined scleral pits as visible indentations of the sclera as seen on fundus photography alone. Scleral tunnels were identified on review of OCT volume scans and were classified based on definitions proposed by Al-Qahtani et al. as linear, hyporeflective channels within the sclera that emerged into either an intact overlying RPE (Type 1), an interruption of the RPE (Type 2), or a full thickness retinal defect (Type 3) [5]. For patients with scleral pits or tunnels, the age and visual acuity from the visit where these abnormalities were first seen were used for statistical comparisons.

The location of scleral tunnels was marked on OCT bscans, and the fovea and center of the optic nerve were used to register the location of tunnels across multimodal imaging and compare variability of their distribution between patients. For purposes of statistical analysis, visual acuities were converted to $\log$ MAR equivalents as previouslydescribed [8]. Univariate analysis was then performed to correlate the presence of scleral pits or tunnels with age, visual acuity, spherical equivalent, and visual field loss (STATA; Stata Statistical Software, Version 14.1; Stata Corporation, College Station, Texas, USA), with $P \leq 0.05$ considered statistically-significant.

\section{Results}

Thirty patients were included in the study. Of these, 21 were affected males (mean age 37.9 years, range 13-72 years) with a median visual acuity of 20/30 (range 20/20 to light perception). Nine female carriers were included (mean age 34.8 years, range 8-69 years). Patient characteristics, including genotypes, are summarized in Table 1.

Eight of the 21 affected males $(38.1 \%)$ had scleral pits visible on fundus photographs, and in all but one patient, these pits were seen in both eyes. Every scleral pit identified was observed to have an emerging vessel within the pit (e.g., Fig. 1). No scleral pits were visualized in any female carriers. The average age of the patients on the visit when their scleral pits were first observable was 45.3 years, compared with 34.0 years for those without scleral pits $(p=$ $0.031)$. Mean visual acuity for those with a scleral pit was logMAR 1.2 (Snellen equivalent about 20/300) versus logMAR 0.2 (Snellen equivalent 20/32) for those without $(p=0.050)$. Of those with available corresponding visual fields, eyes with scleral pits were more likely $(p=0.023)$ to have complete loss of the I2e isopter on Goldmann perimetry $(7 / 15$ eyes $46.7 \%)$ than those without (6/31 eyes, $19.4 \%)$. Similarly, eyes with scleral pits were more likely $(p=0.007)$ to have less than $20^{\circ}$ of central visual field to the V4e isopter (11/15 eyes, $73.3 \%)$ compared with those without pits (9/31 eyes, $29.0 \%)$. Of those with an available refraction, patients with scleral pits were more myopic (average spherical equivalent -3.1 diopters) compared with those without ( -1.4 diopters), but this difference was not statistically-significant $(p=0.085)$. 


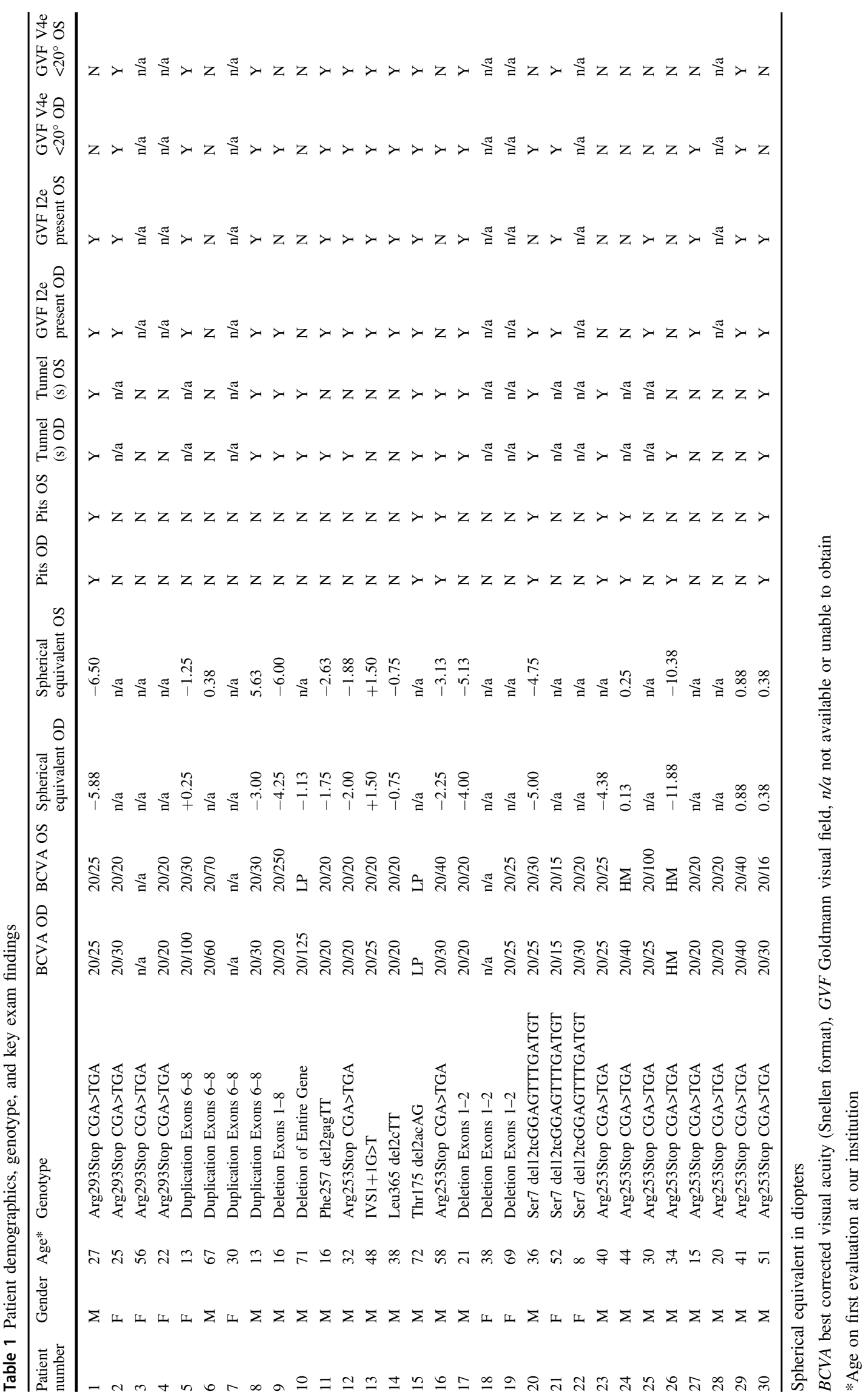




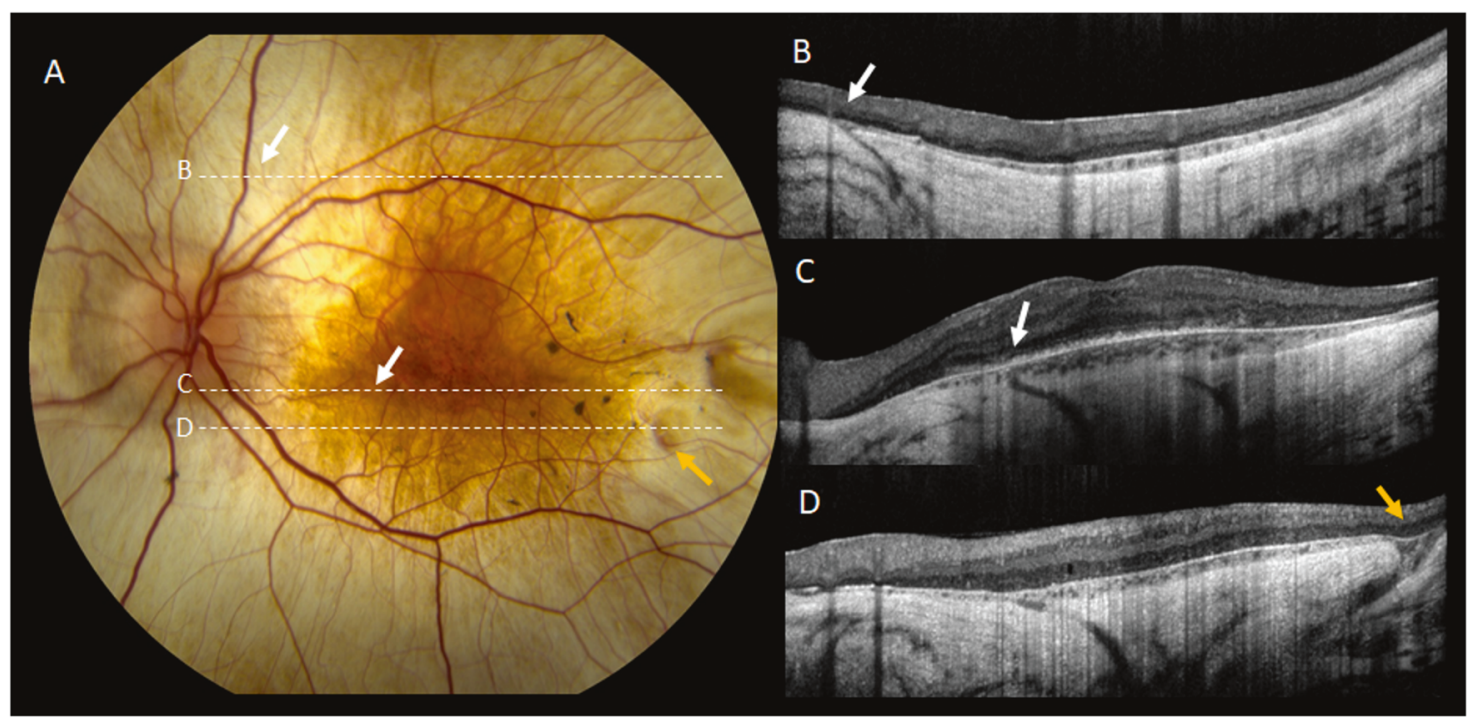

Fig. 1 Examples of scleral tunnels (Patient 1, left eye). Color fundus photograph (a) with dashed horizontal lines corresponding to optical coherence tomography (OCT) raster scans shown on the right (b-d). Arrows demonstrate the location of scleral tunnels shown in cross section in the OCT line scans. A scleral pit with an emerging vessel is seen in a corresponding to the scleral tunnel highlighted in $\mathbf{d}$ (yellow arrows)
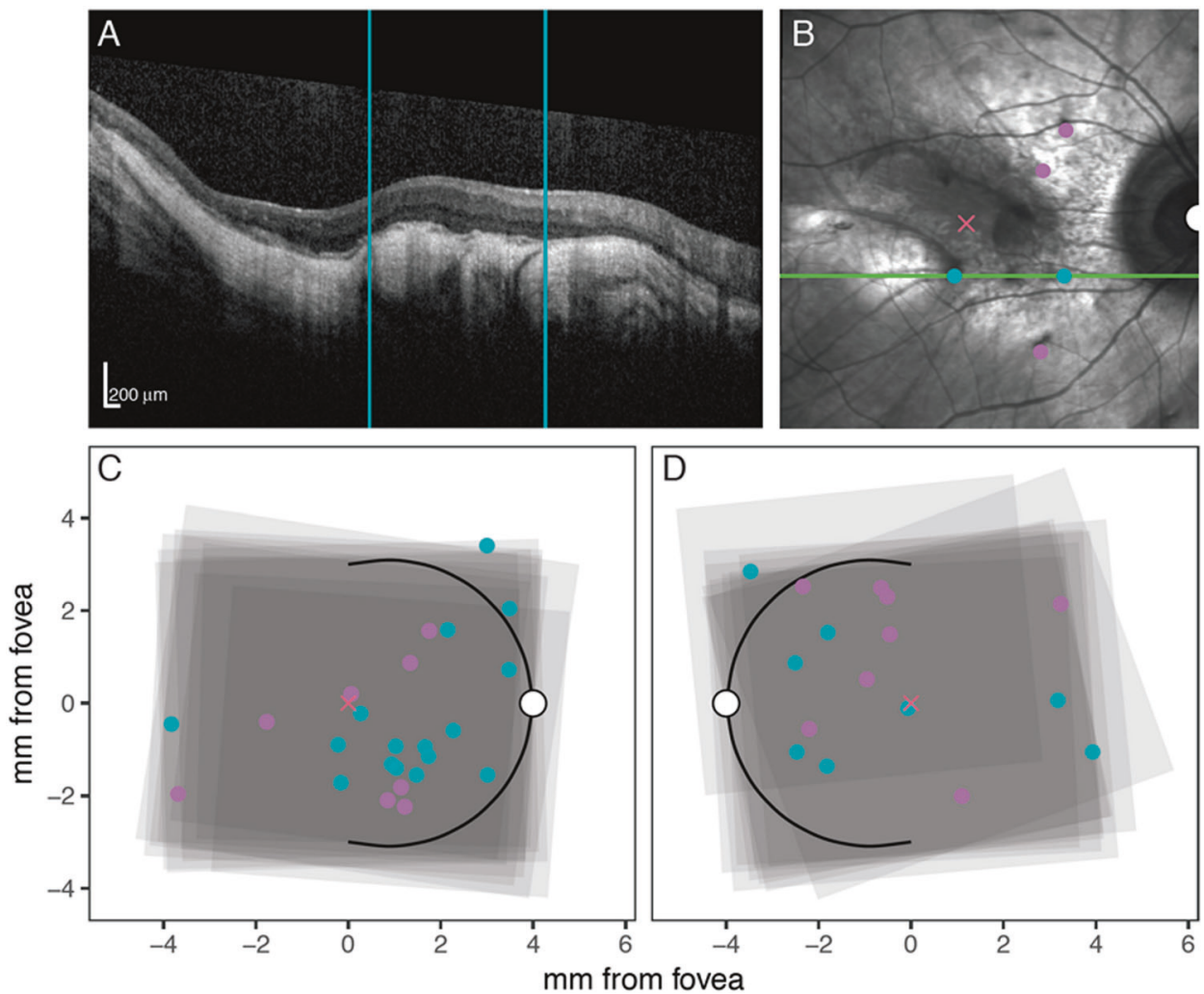
Legend
$\times$ Fovea
Optic Disk
- Type 1 Tunnel
- Type 2 Tunnel

Fig. 2 Localization of scleral tunnels. Optical coherence tomography line scan (a) and corresponding en face reflectance image (b) of the right eye from Patient 10 showing an example of scleral tunnel co-

OCT imaging was available for 19 of the 21 affected male patients. Scleral tunnels were seen in $60.5 \%$ of eyes (23/38) from 13 different male patients $(13 / 19,68.4 \%)$, with localization. $\mathbf{c}$ and $\mathbf{d}$ show the distribution relative to the optic nerve and fovea for all 13 patients with scleral tunnels

an average of 2.65 tunnels per eye. The number of type 1 (33/61 tunnels, 54.1\%) and type 2 tunnels (28/61 tunnels, $45.9 \%$ ) were similar, and no type 3 tunnels were seen. 


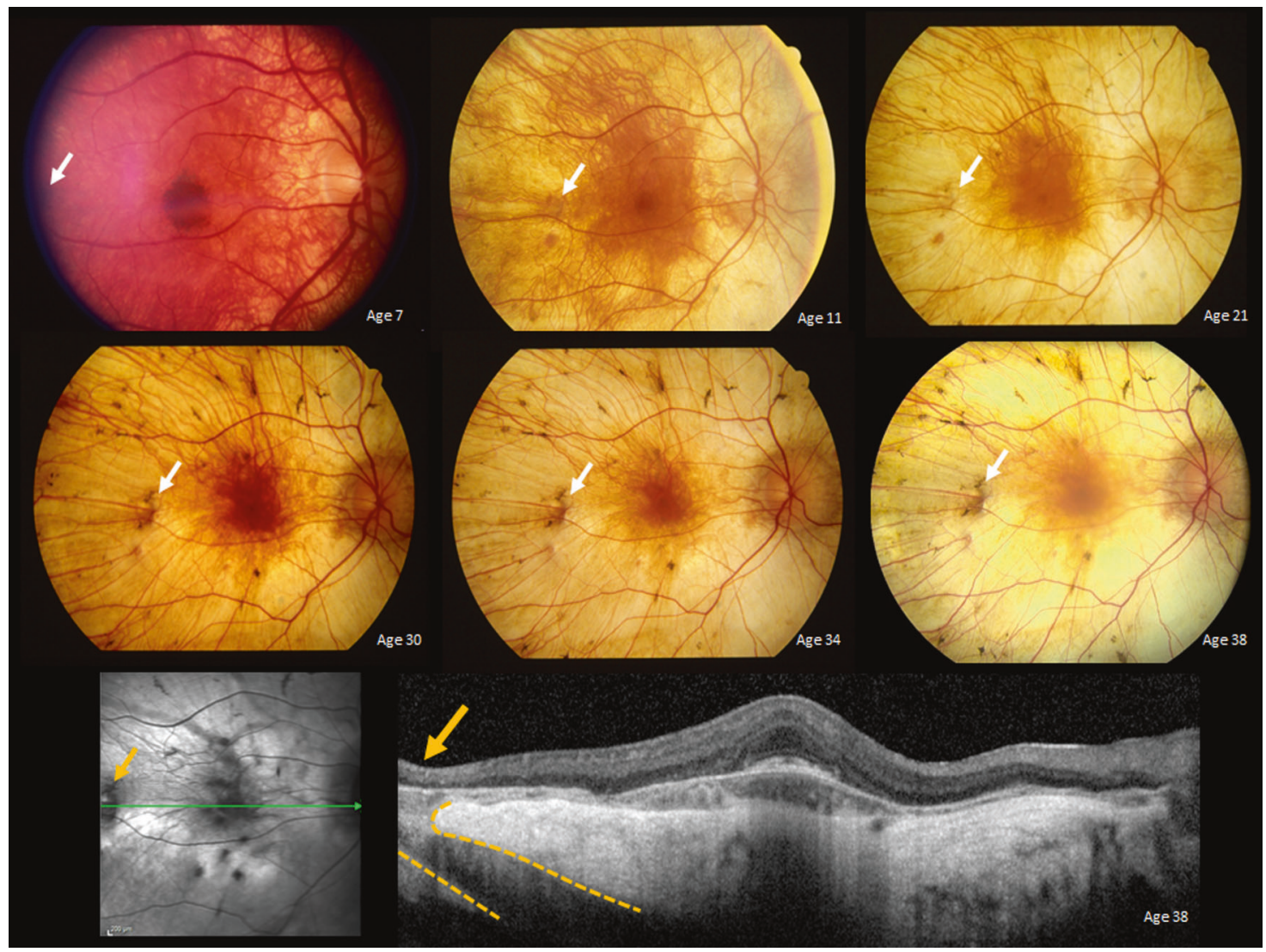

Fig. 3 Longitudinal imaging showing scleral pit formation (Patient 23) over 30 years. Top two rows: color fundus photographs of the right eye, with white arrows showing location of pit development. Bottom

Figure 1 shows examples of type 1 and type 2 tunnels seen in the same patient, including a type 2 tunnel with an associated scleral pit.

When co-localizing tunnel locations across multimodal imaging, all scleral pits seen on fundus photography were found to have a corresponding type 2 scleral tunnel on OCT imaging when available, but some type 2 tunnels were seen on OCT without corresponding scleral pits on fundus photography. All type 1 tunnels were noted, by definition, to have overlying preservation of RPE. No type 1 tunnels were found to have corresponding scleral pits on fundus photography. Figure 2 shows a case example marking the location of scleral tunnels (Patient 10, right eye) as well as composite images summarizing tunnel locations relative to the optic nerve and fovea for all patients.

Eyes with scleral tunnels had poorer mean visual acuity (logMAR 0.64, Snellen equivalent about 20/80) than those without (logMAR 0.26, Snellen equivalent about 20/40), but this difference was not statistically-significant $(p=$ 0.17). Similarly, eyes with scleral tunnels were more myopic (average spherical equivalent -3.4 versus -1.1 diopters), but this difference was not statistically-significant $(p=0.19)$. In eyes with scleral tunnels, visual fields were row: corresponding en face near infrared image (left) and optical coherence tomography line scan (right) showing a type 2 scleral tunnel (yellow arrow and dashed lines)

more likely to have complete loss of the I2e isopter $(9 / 23$ eyes, $39.1 \%$ versus $3 / 15$ eyes, $20.0 \% ; p=0.33$ ) and less than $20^{\circ}$ of central field to the V4e isopter $(11 / 23,47.8 \%$ versus $9 / 15$ eyes, $60.0 \%$ ), but these differences were also not statistically-significant $(p=0.28)$.

Longitudinal fundus photography was available for 10 of the 30 patients, including eight affected males and two carriers. Average length of follow-up was 12.8 years (range 4.0-30.5 years). Among the eight affected males, scleral pits were seen in four patients $(50.0 \%)$. In each of these patients, the scleral pits were noted only on follow-up imaging, with no definite scleral pits seen on the photographs from their initial visits. Figure 3 shows an example of longitudinal fundus photography demonstrating scleral pit formation over 30 years of follow-up (Patient 23). Longitudinal OCT was available for seven male patients, with average length of follow-up of 5.0 years (range 1.0-7.2 years). Two individuals, Patient 8 and 17, were noted to have development of new, type 2 scleral tunnels on longitudinal OCT imaging over 10 and 8 years, respectively. Figure 4 demonstrates the development of a type 2 scleral tunnel over the course of 10 years (Patient 8 ). No scleral tunnels were observed to convert between tunnel types 
Fig. 4 Scleral tunnel development over 10 years (Patient 8, left eye). Left column: near infrared en face reflectance images showing location of corresponding line scan locations (arrowed). Right column: registered optical coherence tomography line scans demonstrating development of a type 2 scleral tunnel (arrowed)

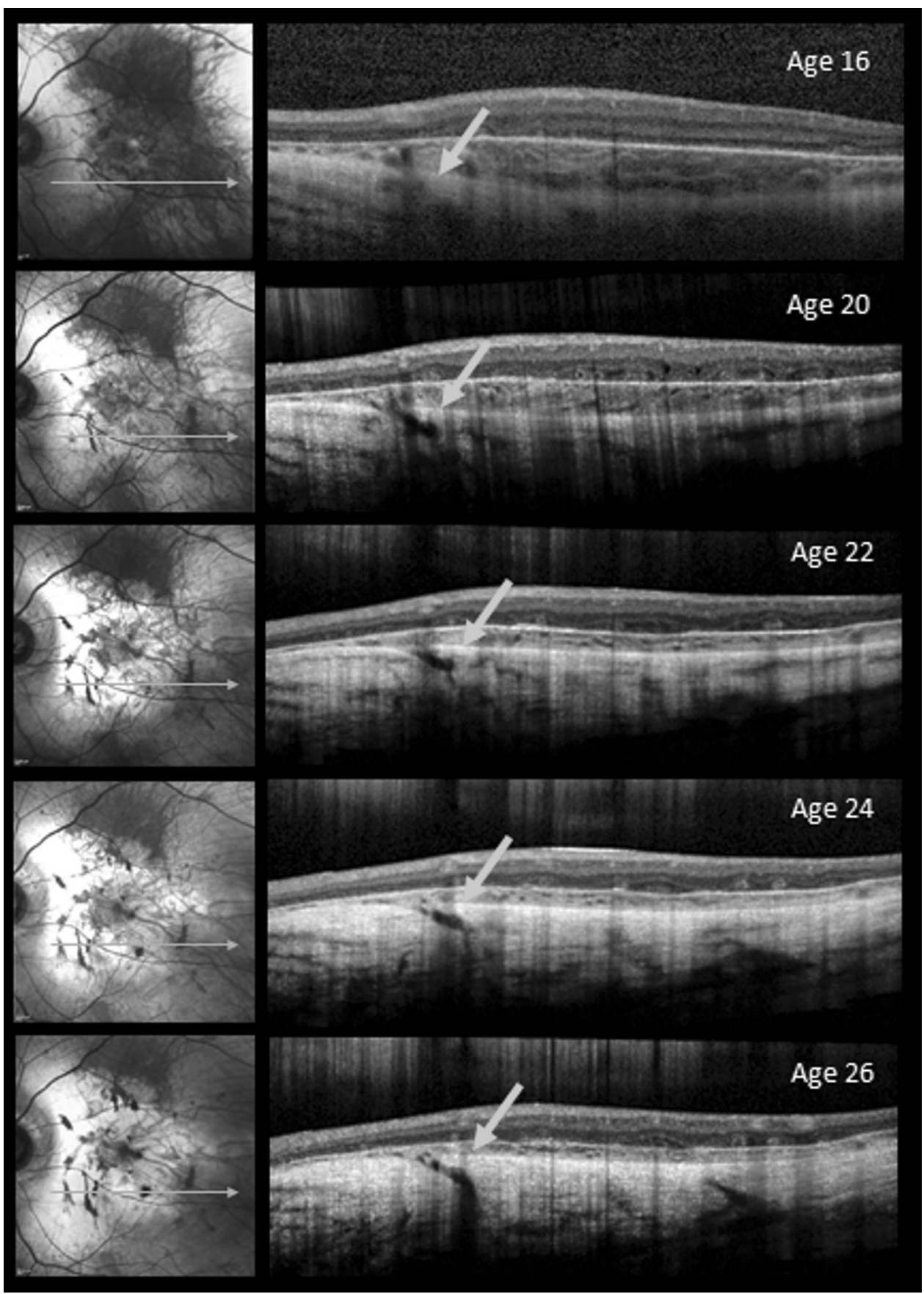

(e.g., from type 1 to type 2) over the course of follow-up imaging for any patients in our study.

\section{Discussion}

This study demonstrates several important findings that expand our understanding of scleral pits and tunnels, which have only recently been reported as a clinical feature of choroideremia $[5,6]$. Specifically, we show that scleral pits and tunnels in choroideremia are not congenital but rather secondary to progressive degeneration, as evidenced by their development over time as seen on longitudinal fundus photography and OCT imaging. We show that scleral pits are associated with older age and more advanced disease, including poorer visual acuity and more severe visual field loss. In contrast, we demonstrate that scleral tunnels are relatively common, are not always associated with scleral pits, and can be seen in eyes with less severe disease. Finally, we describe the regional distribution of scleral tunnels in the macula and provide evidence of their association with the short and long posterior ciliary arteries. 
Initial reports describing scleral "pits" and "tunnels" as novel findings in choroideremia used these two terms interchangeably and did not distinguish between their identification on either fundus photography or OCT imaging $[5,6]$. For the sake of clarity and to better elucidate any potential differences in pathophysiology and associations with disease severity, we defined scleral "pits" as those seen on fundus photography because these focal excavations are evident even without OCT visualization of the underlying choroid and sclera, and "tunnels" as those seen on OCT imaging where cross-sectional imaging allows visualization of their extent within the sclera. Using these definitions, the prevalence of scleral pits $(38.1 \%)$ and tunnels $(60.5 \%)$ in our affected male cohort were similar to those reported by van Schuppen et al., who noted scleral pits on fundus photography in $33.3 \%$ and tunnels on OCT in $61.9 \%$ of their 21 affected male participants [6]. Al-Qahtani et al. analyzed the presence of scleral ectasias in a multicentre cohort of 36 affected males and found scleral tunnels on OCT in $52.8 \%$ of eyes. They further associated the presence of scleral pits and tunnels together with older age, greater myopic refractive error, and poorer visual acuity [5].

The results of our study are supportive of scleral pits as a marker of disease severity, as patients with scleral pits seen on fundus photography were older, had poorer visual acuity, and more severe visual field loss. However, unlike scleral pits, scleral tunnels are relatively common and may be seen earlier in the disease course. All scleral pits seen on fundus photography in our study possessed corresponding scleral tunnels when evaluated cross-sectionally by OCT imaging, but not all scleral tunnels were associated with scleral pits. Although patients with scleral tunnels also tended to be older, have poorer visual acuity, and more severe visual field loss than those without scleral tunnels, none of these comparisons were statistically-significant. Taken together, scleral pits have a clearer association with advanced disease and may be a more reliable marker of disease severity than scleral tunnels. Because both scleral pits and tunnels appear very slow to develop or change (on the order of years) neither may be ideal markers to track disease progression for clinical trials.

Previous studies describing scleral pits and tunnels in choroideremia have lacked analysis of longitudinal imaging to demonstrate whether these scleral abnormalities are congenital or related to progressive degeneration. In this study, we show the development of scleral pits and tunnels over time using longitudinal imaging, as highlighted in Figs. 3 and 4. The pathogenesis of scleral pits and tunnels remains unknown. One contributor to their formation, however, may be scleral thinning related to axial myopia, as focal scleral ectasias have been described previously in pathologic myopia [9]. Al-Qahtani et al. found that choroideremia patients with scleral tunnels had a greater degree of myopia than those without [5]. In our choroideremia cohort, most patients had only moderate myopia. Those with scleral pits and tunnels were more myopic than those without pits or tunnels, but this difference was not statistically-significant, and our study may have been underpowered to detect a difference.

Although scleral pits can be seen in other conditions such as pathologic myopia, the high prevalence of scleral pits and tunnels in our choroideremia cohort suggests that the formation of these scleral ectasias requires more than just scleral thinning due to myopia and may involve focal weaknesses in the sclera, including at the site of perforating choroidal vasculature $[5,6]$. In our cohort, every scleral pit visualized on fundus photography had a choroidal vessel emerging from the pit. Some scleral tunnels did not have a visible choroidal vessel, but we suspect this was because of an inability to visualize these deeper choroidal vessels due to preserved overlying RPE (e.g., Fig. 1b, c) rather than true lack of association. We did not have corresponding indocyanine, fluorescein, or OCT angiography available for the patients included in the study to co-localize choroidal vasculature deep to preserved RPE. Therefore, to demonstrate the location of scleral tunnels relative to the optic nerve and fovea, we registered their positions in the posterior pole across OCT imaging as shown in Fig. 2. The relative distribution of scleral tunnels in our cohort are consistent with published literature on the insertion points of both the long and short posterior ciliary arteries [10]. While these scleral passages are present in normal retinal anatomy, serving as conduit for the ciliary arteries, they become visible with overlying retinal and choroidal degeneration. Large scleral tunnels were seen temporal to the fovea, where long, oblique, hyporeflective channels in the sclera could be seen following the path of the long posterior ciliary nerve and artery (e.g., Fig. 1d). Smaller and more focal scleral pits tended to be observed in and around the macula and correspond to the insertion of the short posterior ciliary arteries. Overall, these data suggest an association of scleral pits and tunnels with normal choroidal vascular anatomy but also provide evidence to suggest degeneration around the short and long posterior ciliary arteries as contributory to their formation in choroideremia.

Surprisingly little exists in the literature regarding the role of choroidal vasculature in the pathophysiology of choroideremia, and the role of the ciliary arteries in particular has not previously been explored. Choroidal vascular defects have been postulated as the cause of choroideremia since the initial description by Mauthner in 1872 [11], and subsequent human histopathologic reports have supported this theory [12-14]. Although it is reasonable to hypothesize that ciliary artery attenuation and tunnel formation are the proximal events leading to overlying RPE and photoreceptor loss, the presence of outer retinal degeneration and 
RPE loss in some eyes where no scleral tunnels are seen argues against ciliary artery involution as the primary defect. Moreover, in each case where scleral tunnel formation was observed during the course of follow-up in our study, the tunnel formed under areas of existing chorioretinal degeneration (e.g., Fig. 4). These findings are in line with recent evidence suggesting that the photoreceptors and RPE may degenerate independently, with underlying choroidal loss as a subsequent event. For example, numerous histopathologic studies, including in female carriers, have demonstrated photoreceptor degeneration in the absence of or in conjunction with RPE degeneration $[15,16]$, with choroidal loss seen only under areas of severe photoreceptor/RPE loss [17]. Similarly, mouse models selectively ablating $C H M$ in the photoreceptors or RPE have shown that these layers can degenerate independently [18, 19]. Most recently, multimodal imaging including OCT with angiography has been used to demonstrate that RPE degeneration precedes loss of the photoreceptors or choriocapillaris [20].

In our study, all scleral pits were found to have a corresponding type 2 tunnel on OCT imaging. We believe that scleral pits represent superficial scleral excavations due to the loss of RPE and disruption of Bruch's membrane, allowing the remaining neuroretinal tissue to sink into the anterior-most aspect of a preexisting scleral tunnel. Given the role of RPE in choroidal development and maintenance [21-24], degeneration of the RPE directly overlying the ciliary arteries may be the key event in scleral pit and tunnel formation. This pathologic mechanism would reconcile the discord between the initial theories of choroidal loss as proposed by Mauthner with more recent evidence of gradual, progressive outer retinal and choroidal loss. In other words, choroideremia involves a slowly progressive degeneration of the photoreceptors, RPE, and choroid, in relatively independent fashion. However, once the RPE overlying an inserting ciliary artery degenerates, this may result in secondary involution of the underlying ciliary artery, scleral tunnel formation, and collapse of the overlying choroidal lobular unit, accounting for the discrete, scalloped areas of chorioretinal atrophy seen clinically. Attenuation or involution of the posterior ciliary artery may then result in pit formation and surrounding scleral thinning. Further study utilizing wide-field longitudinal multimodal imaging including OCT angiography or indocyanine green angiography may be useful in elucidating the temporal relationship between scleral tunnel formation, ciliary artery involution, and photoreceptor, RPE, and choriocapillaris loss.

To the best of our knowledge, this study is the first to utilize longitudinal imaging to visualize the development of scleral pits and tunnels and provide insight into their formation over time. Our study was retrospective, with varying availability of testing as well as frequency and duration of follow-up across our cohort. Most patients
$(60.5 \%)$ in our study had scleral tunnels visible on OCT, with a similar number of type 1 and type 2 tunnels observed. We did not visualize any type 3 tunnels in our study, perhaps because patients with type 3 tunnels may have more severe disease and be more difficult to image with OCT due to poor visual acuity or fixation, or media opacity. Patients with choroideremia often have patchy peripheral visual field loss. Rather than quantifying the extent of field loss, we chose binary measures of global severity (complete absence of $\mathrm{I} 2 \mathrm{e}$ isopter) and central preservation $\left(>20^{\circ}\right.$ remaining $\left.\mathrm{V} 4 \mathrm{e}\right)$. It is possible that quantitative measures of retinal function, including area analysis of visual field area or measurement of ERG amplitudes, may provide more sensitive markers to correlate to the presence of scleral ectasias. We did not have axial length measurements available for patients in our cohort, and future studies may investigate the association between axial length and the presence of scleral pits or tunnels. The degree of chorioretinal degeneration in choroideremia typically allows adequate visualization through the choroid, but visualization of tunnels and their course through the sclera may be enhanced utilizing a longer wavelength laser such as with swept-source OCT imaging [25]. The use of swept-source imaging may also allow characterization of pathologic changes outside the central macula, and correlation of structural findings on longitudinal OCT with vascular changes on angiography may improve our understanding of the relationship between chorioretinal degeneration, choroidal vascular loss, and scleral tunnel formation over time.

\section{Summary}

\section{What was known before}

- Peculiar scleral ectasias, referred to as scleral pits and tunnels can be seen in patients with choroideremia.

- The presence of scleral pits has been associated with older age and poorer visual acuity, but little is known regarding their etiology.

\section{What this study adds}

- Demonstrates that scleral pits are associated with older age, worse visual acuity, and more severe visual field loss.

- Provides longitudinal imaging to show that scleral pits are a result of progressive atrophic degenerative (i.e., not congenital).

- Shows the location of the scleral tunnels in the macula, providing evidence that they occur due to tissue 
degeneration around the inserting posterior ciliary arteries.

Funding This research is supported by University of Iowa, Institute for Vision Research.

\section{Compliance with ethical standards}

Conflict of interest The authors declare that they have no conflict of interest.

Publisher's note Springer Nature remains neutral with regard to jurisdictional claims in published maps and institutional affiliations.

\section{References}

1. Coussa RG, Traboulsi EI. Choroideremia: a review of general findings and pathogenesis. Ophthalmic Genet. 2012;33:57-65.

2. Simunovic MP, Jolly JK, Xue K, et al. The spectrum of CHM gene mutations in choroideremia and their relationship to clinical phenotype. Investig Ophthalmol Vis Sci. 2016;57:6033-9.

3. Cremers FP, van de Pol DJ, van Kerkhoff LP, Wieringa B, Ropers $\mathrm{HH}$. Cloning of a gene that is rearranged in patients with choroideraemia. Nature. 1990;347:674-7.

4. Lee SY, Yu WK, Lin PK. Large gene deletion and changes in corneal endothelial cells in a family with choroideremia. Investig Ophthalmol Vis Sci. 2015;56:1887-93.

5. Al-Qahtani AA, Ba-Ali S, Alabduljalil T, et al. Scleral pits in choroideremia: implications for retinal gene therapy. Retina. 2018;38:1725-30.

6. van Schuppen SM, Talib M, Bergen AA, et al. Long-term followup of patients with choroideremia with scleral pits and tunnels as a novel observation. Retina. 2018;38:1713-24.

7. Stone EM, Andorf JL, Whitmore SS, et al. Clinically focused molecular investigation of 1000 consecutive families with inherited retinal disease. Ophthalmology. 2017;124:1314-31.

8. Roberts MF, Fishman GA, Roberts DK, et al. Retrospective, longitudinal, and cross sectional study of visual acuity impairment in choroideraemia. Br J Ophthalmol. 2002;86:658-62.

9. Pedinielli A, Souied EH, Perrenoud F, Leveziel N, Caillaux V, Querques $\mathrm{G}$. In vivo visualization of perforating vessels and focal scleral ectasia in pathological myopia. Investig Ophthalmol Vis Sci. 2013;54:7637-43.
10. Hayreh SS. Posterior ciliary artery circulation in health and disease: the Weisenfeld lecture. Investig Ophthalmol Vis Sci. 2004:45:749-57. 748

11. Mauthner L. Ein Fall von Choroideremia. Ber d Nat-med, Ver Innsbr 1872;11:191.

12. Cameron JD, Fine BS, Shapiro I. Histopathologic observations in choroideremia with emphasis on vascular changes of the uveal tract. Ophthalmology. 1987;94:187-96.

13. Ghosh M, McCulloch JC. Pathological findings from two cases of choroideremia. Can J Ophthalmol. 1980;15:147-53.

14. Mc CJ. The pathologic findings in two cases of choroideremia. Trans Am Acad Ophthalmol Otolaryngol. 1950;54:565-72.

15. Bonilha VL, Trzupek KM, Li Y, et al. Choroideremia: analysis of the retina from a female symptomatic carrier. Ophthalmic Genet. 2008;29:99-110.

16. Flannery JG, Bird AC, Farber DB, Weleber RG, Bok D. A histopathologic study of a choroideremia carrier. Investig Ophthalmol Vis Sci. 1990;31:229-36.

17. Syed N, Smith JE, John SK, Seabra MC, Aguirre GD, Milam AH Evaluation of retinal photoreceptors and pigment epithelium in a female carrier of choroideremia. Ophthalmology. 2001;108:711-20.

18. Tolmachova T, Anders R, Abrink M, et al. Independent degeneration of photoreceptors and retinal pigment epithelium in conditional knockout mouse models of choroideremia. J Clin Investig. 2006;116:386-94.

19. Tolmachova T, Wavre-Shapton ST, Barnard AR, MacLaren RE, Futter CE, Seabra MC. Retinal pigment epithelium defects accelerate photoreceptor degeneration in cell type-specific knockout mouse models of choroideremia. Investig Ophthalmol Vis Sci. 2010;51:4913-20.

20. Jain N, Jia Y, Gao SS, et al. Optical coherence tomography angiography in choroideremia: correlating choriocapillaris loss with overlying degeneration. JAMA Ophthalmol. 2016;134:697-702.

21. Korte GE, Reppucci V, Henkind P. RPE destruction causes choriocapillary atrophy. Investig Ophthalmol Vis Sci. 1984;25:1135-45.

22. Marneros AG, Fan J, Yokoyama Y, et al. Vascular endothelial growth factor expression in the retinal pigment epithelium is essential for choriocapillaris development and visual function. Am J Pathol. 2005; 167:1451-9.

23. Saint-Geniez M, Kurihara T, Sekiyama E, Maldonado AE, D'Amore PA. An essential role for RPE-derived soluble VEGF in the maintenance of the choriocapillaris. Proc Natl Acad Sci USA 2009; 106:18751-6.

24. Summers JA. The choroid as a sclera growth regulator. Exp Eye Res. 2013;114:120-7.

25. Cunningham CM, Critser DB, Han IC. Swept-source OCT of a scleral tunnel in choroideremia. Ophthalmology. 2018;125:806-806. 